\title{
A survey of referrers' satisfaction with a regional forensic psychiatric service: what do they want?
}

\author{
AIMS AND METHOD \\ The perceptions and expectations by \\ referrers of assessments performed \\ by a medium secure unit were \\ examined in order to ascertain areas \\ for possible improvement. All \\ referrals to two teams at the North \\ West Thames Regional Secure Unit \\ were monitored over a 6 -month \\ period. A self-report questionnaire \\ was sent to each referrer, in cases
}

\author{
where an assessment and forensic \\ report had been completed.
RESULTS
Assessments and forensic reports were completed (and questionnaires sent to referrers) in $63 \%$ of total referrals (32 out of 51). The response rate to the questionnaire was $81 \%$ ( 26 out of 32). Many referrers wanted the assessments and report to be

\begin{abstract}
completed in 2 weeks. Most referrers were satisfied with the quality of the report received and the majority were happy with the risk assessment.

\section{CLINICAL IMPLICATIONS}

Referrers want forensic assessments to be of a high quality and to be performed quickly. Recommendations for service development are suggested.
\end{abstract}

Following the recommendations of the Butler (Home Office \& Department of Health and Social Security, 1975) and Reed (Department of Health \& Home Office, 1992) reports, there has been a rapid development and expansion of forensic psychiatry, with an increase in the number of medium secure beds, and subsequently, there has been a rise in the number of requests for forensic psychiatric assessments. Despite the increasing number of referrals, it is not always possible to identify clearly the specific reason for a referral and the particular issues which the referrer would like the forensic service to address. In general, there is no formal mechanism for assessing the level of satisfaction of referrers with the nature and quality of medium secure services, nor the degree to which services correspond to their expectations.

Studies have investigated aspects of forensic care (Sighal, 1994; Mendelson, 1993), but there has been no specific focus on the views of referrers regarding the advantages and shortcomings of the forensic mental health service.

Phillips et al (1996) discussed the results of a patient satisfaction survey undertaken at one medium secure unit. The authors emphasised the importance of continuing to seek the views of the 'consumers' of forensic mental health services as one strand of the planning process for future services.

\section{Method}

Our survey was conducted at the North West Thames regional forensic service, which covers a population of approximately 3.5 million. There are 123 medium secure beds with eight sector teams, plus a remand bed service. Each team is multi-disciplinary with a consultant plus junior doctors; all have psychology, occupational therapy and community psychiatric nurse (CPN) input. Unlike some other medium secure units, there is a welldeveloped local secure service on the same site that comprises 82 low secure beds and 52 rehabilitation and pre-discharge beds.

The referrals to two sector teams, covering an urban catchment area with a total population of 345000 , were monitored over a 6-month period (March to August 1999). We selected three main aspects of referrer satisfaction for evaluation regarding:

(1) time taken to receive written acknowledgement of the referral;

(2) time taken to receive the final completed report;

(3) quality of the report.

These were evaluated through a self-report questionnaire. The referrer was invited to make comments or suggestions where they were dissatisfied with any aspect of the assessment.

\section{Results}

Fifty-eight referrals were received, of which 51 were clinical and seven medico-legal. Only the clinical referrals were included in the survey.

Sixty-five per cent (33 out of 51) of the referrers requested patient management advice and risk assessment; $25 \%$ (13 out of 51) asked for transfer of a patient to a medium secure unit.

Psychiatrists were allocated 61\% (31 out of 51) of the referrals and $35 \%$ (18 out of 51) passed to psychologists. Two referrals were not allocated. Of the 49 referrals allocated, reports were presented for only 32 since only $28 \%$ of referrals allocated to psychologists resulted in a report. The reasons for reports not being written included patients being referred onto another service for assessment or treatment, patients remaining in assessment or verbal feedback being given to the referrer.

Over the 6-month period, 32 (63\%) reports and questionnaires were sent out and 26 were returned, giving a response rate of $81 \%$ (26 out of 32 ). 


\section{Referrer satisfaction}

The time taken to receive the final report was regarded as satisfactory by $58 \%$ (15 out of 26 ) of referrers; $42 \%$

(11 out of 26 ) of referrers were not satisfied with $82 \%$ of this group expecting to receive a report within 3 weeks. Overall, the unit only sent out $26 \%$ of reports within 4 weeks of referral (Table 1).

\section{Referrers' comments}

Referrers suggested that regular meetings between community mental health teams and the forensic service and more communication to discuss recommendations in complex cases could improve the service. They also asked for doctors and nurses to receive further training on key issues of risk assessment and management and, finally, for a quicker response time for assessments.

\section{Discussion}

This study exclusively examines clinical referrals to the service. Generally, referrers were satisfied with the quality of reports, including advice on management, risk assessment and the clarity of opinion and recommendations.

In 13 cases, the referrer requested a patient be admitted to the medium secure unit. Two patients were admitted to the unit; a further three were admitted to the local secure service. This may seem a small proportion of the requests made and it is recognised that this service has a high threshold for admission. It is also recognised that the North West Thames service refers relatively few patients to a special hospital and this contributes to a larger number of more complex cases being managed by the service. One-third of patients have been in the medium secure unit for over 2 years and $10 \%$ for more than 5 years. In addition, many in-patients were directly discharged to the community rather than being returned to local services.

A substantial number of referrers requested advice on management of either an in-patient or an out-patient and a significant number asked specifically for a risk assessment. It is likely that there is an increase in clinical referrals for risk assessment. Requests for such assessments are common to all medium secure services, although there is a regional variation in the style of service provided. Because of findings in recent homicide

\section{Table 1. Referrer satisfaction with report received}

\begin{tabular}{lc} 
Report & Referrer satisfaction (\%) \\
\hline $\begin{array}{l}\text { contains all relevant information } \\
\text { gives a clear opinion and } \\
\text { recommendations }\end{array}$ & 88 (23 out of 26$)$ \\
$\begin{array}{l}\text { addresses concerns of referrer } \\
\text { gives advice on risk assessment and } \\
\text { management }\end{array}$ & 81 (21 out of 26$)$ \\
\end{tabular}

inquiries such as Clunis (Ritchie et al, 1994), clinicians are more anxious about the treatment and rehabilitation of potentially dangerous patients. Current psychiatric ethos places considerable emphasis on the importance of risk assessment and clinicians are more inclined to request a second opinion from forensic psychiatric services in the cases of offenders who are mentally ill, or patients with a history of, or potential for, violence.

This survey was designed to be multi-disciplinary, including all practitioners participating in forensic assessments. Many disciplines, including nurses, art and music therapists, psychotherapists and occupational therapists, play an important role in the rehabilitation of offenders who are mentally ill. However, the survey shows that all referrals were allocated to either doctors or psychologists to the exclusion of the others. One explanation is limited staff numbers, but these results may also reflect an expectation of referrers to receive a medical or psychological opinion. Medical responsibility for the recommendations made is an inevitable factor that motivates doctors to see patients themselves. However, it is interesting to note that the suggestion to move towards a multi-disciplinary model was made frequently by referrers.

We suggest that multi-disciplinary assessment by practitioners from different core disciplines is good clinical practice. It offers a broader perspective on a case and an opportunity for mutual staff development. In our survey, the low proportion of reports written by psychologists after an assessment makes it more difficult to assess their work in terms of referrer satisfaction. We suggest it would be valuable to undertake a further study that specifically addresses the question of satisfaction with the service offered by psychologists compared with that given by psychiatrists. It should also address whether referrers feel that the production of a formal report (in addition to a written clinical record) is an essential component of the service.

This raises an important question about surveys of this nature. Should they examine solely medical or multidisciplinary practice? Glover (1990) emphasises the fact that, despite the importance of multi-disciplinary teamwork both within psychiatric services and at an interagency level, a multi-disciplinary approach will complicate audit considerably as poor results may reflect a difference in practice between disciplines.

\section{Implications for the service}

Referrers are concerned about the time taken to receive a report; here there is a considerable gap between what they expect from a forensic psychiatric service and what is provided in practice.

How useful a forensic psychiatric report is to the referring clinician may depend to a large extent on the time taken to produce it, for example, whether or not it is available before a Care Programme Approach meeting or a mental health review tribunal. Referring clinicians may also need a rapid risk assessment and/or management original papers 
advice following a dangerous incident on a ward or in the community.

However, there is a conflict between the quality

original

papers of a report and the time needed for its preparation. A thorough assessment may require considerable investigation, making it difficult by providing a high-quality report in limited time to meet the requirements of the referrer.

Closer links between general psychiatric and medium secure services have much to offer. A regular meeting with the referring team allows discussion of particular cases and addresses expectations of what can be realistically offered. Better communication between referrer and assessor at each point of the assessment facilitates sharing of important information. It also provides mutual education in the needs of the referrer and education on risk assessment. A similar advantage is offered by Joint assessments performed by members of both the referring and assessing teams and offers a similar advantage.

Finally, this study demonstrates the usefulness of obtaining feedback in order to develop services and to meet the perceived needs of the referrers. Evaluating services this way is becoming an essential part of NHS practice and it is one of the main structures that underpins clinical governance (Lugon \& Secker-Walker 1999).

\section{Acknowledgements}

We wish to thank Ms Roz Brown (Referrals Manager) and Ms Penny Steed (Directorates Development
Co-ordinator) at West London Mental Health NHS Trust for assisting us in collating the data for this survey.

\section{Declaration of interest}

None.

\section{References}

DEPARTMENT OF HEALTH \& HOME OFFICE (1992) Review of Health and Social Services for Mentally Disordered Offenders and Others Requiring Similar Services (Reed Report) (Cm. 2088). London: HMSO.

GLOVER, R. G. (1990) The audit of mental health services. Quality Assurance in Health Care, 2, 181-188.

HOME OFFICE \& DEPARTMENT OF HEALTH AND SOCIAL SECURITY (1975) Report of the Committee on Mentally Abnormal Offenders (Butler Report) (Cmnd 6244). London: HMSO.

LUGON, M. \& SECKER-WALKER, J. (1999) Clinical Governance. Making it Happen. London: Royal Society of Medicine Press.

MENDELSON, E. F. (1993) Underlying patterns of practice in a regional forensic psychiatric service. Medicine Science and the Law, 33, 55-62.

PHILLIPS, C., BURNARD, P. \& MORRISON, P. (1996) Quality in provision of forensic psychiatric services: room for improvement. International Journal of Health Care QualityAssurance, 9, 26-32.

RITCHIE, J., DICK, D. \& LINGHAM, R. (1994) Report of the Inquiry into the Care and Treatment of Christopher Clunis (Cmnd 9543). London: HMSO.

SIGHAL, S. (1994) Audit of forensic psychiatric discharge summaries. Medicine, Science and the Law, 34 $167-169$.

Maria Papanastassiou Specialist Registrar in Psychiatry, Paterson Centre for Mental Health, St Mary's Hospital, London, Sean Roche Specialist Registrar in Psychiatry, University College Hospital PsychiatricTraining Scheme, London, *John Boyle Consultant Adult Psychiatrist, Bexleyheath Centre, 4 Emerton Close, Bexleyheath DA6 8DX， Richard Baxter Consultant Forensic Psychiatrist, Paul Chesterman Consultant Forensic Psychiatrist, NorthWest Thames Regional Forensic Psychiatric Service, Southall, Middlesex 